\title{
Medida de $g$ com a placa Arduino em um experimento simples de queda livre
}

\author{
Measurement of $g$ with an Arduino microcontroller in a simple free fall experiment \\ H. Cordova ${ }^{1}$, A. C. Tort*1 \\ ${ }^{1}$ Mestrado Profissional em Ensino de Física, Instituto de Física, Universidade Federal do Rio de Janeiro, Rio de \\ Janeiro, RJ, Brasil
}

Recebido em 8 de dezembro de 2015. Aceito em 4 de janeiro de 2016

\begin{abstract}
Um modo simples de medir a aceleração da gravidade $g$ no laboratório de física do ensino médio e de física básica no ensino universitário com um microcontrolador da família Arduíno é proposto. Resultados experimentais com um erro relativo de $0,1 \%$ são apresentados e comparados com o valor local de $g$ medido pelo Observatório Nacional, Rio de Janeiro.
\end{abstract}

Palavras-chave: aceleração da gravidade, queda livre, Arduino.

A simple way of measuring the terrestrial acceleration $g$ in the basic lab that makes use of the Arduino microcontroller is proposed. Experimental results with a relative error of $0,1 \%$ were obtained and compared with the local value of $g$ as measured by the Brazilian National Observatory at Rio de Janeiro.

Keywords: gravitational accleration; free fall; Arduino.

\section{Introdução}

Um dos muitos modos possíveis de medir a aceleração da gravidade $g$ é deixar cair verticalmente um corpo a partir de uma altura predeterminada $h$ e medir a duração do seu tempo de queda. Como a velocidade inicial é nula, segue que:

$$
g=\frac{2 h}{t^{2}},
$$

onde $t$ é a duração da queda. Embora a Eq.(1) possa ser introduzida nos primeiros estágios do ensino da cinemática e seja o fundamento de uma das maneiras mais simples de medir $g$, a rapidez com que a queda livre acontece pode tornar o experimento frustante para um novato. É possível melhorar os resultados por meio de circuitos eletrônicos capazes de determinar intervalos de tempo com pelo menos 1/10 de milissegundo de resolução, veja por exemplo [1], mas estas técnicas são mais apropriadas para os laboratórios didáticos avançados dos cursos de graduação. Por outro lado, o uso cada vez

*Endereço de correspondência: tort@if.ufrj.br mais difundido dos modernos microcontroladores de baixo custo, como por exemplo, a família de microcontroladores ou placas Arduíno [2 6], capazes de medir intervalos de tempo na faixa de mili e microssegundos permite obter resultados perfeitamente aceitáveis no laboratório de física do ensino médio e de física básica no ensino universitário, mesmo que em uma primeira abordagem, por conveniência pedagógica, desprezemos os efeitos da resistência do ar e outros efeitos espúrios. Nossa prática experimental nos diz que enquanto $h$ for uma altura inferior a 1 metro tais efeitos podem ser desconsiderados em uma primeira abordagem. Nas próximas seções discutiremos como isto pode ser feito.

\section{Arranjo experimental}

O arranjo experimental é mostrado na Figura 1. Uma pequena esfera de aço de $9 \mathrm{~mm}$ de diâmetro está inicialmente presa entre duas alavancas metálicas, uma fixa e outra móvel. Estas duas alavancas fazem parte do sensor superior, que também funciona como plataforma de lançamento da esferinha, veja 


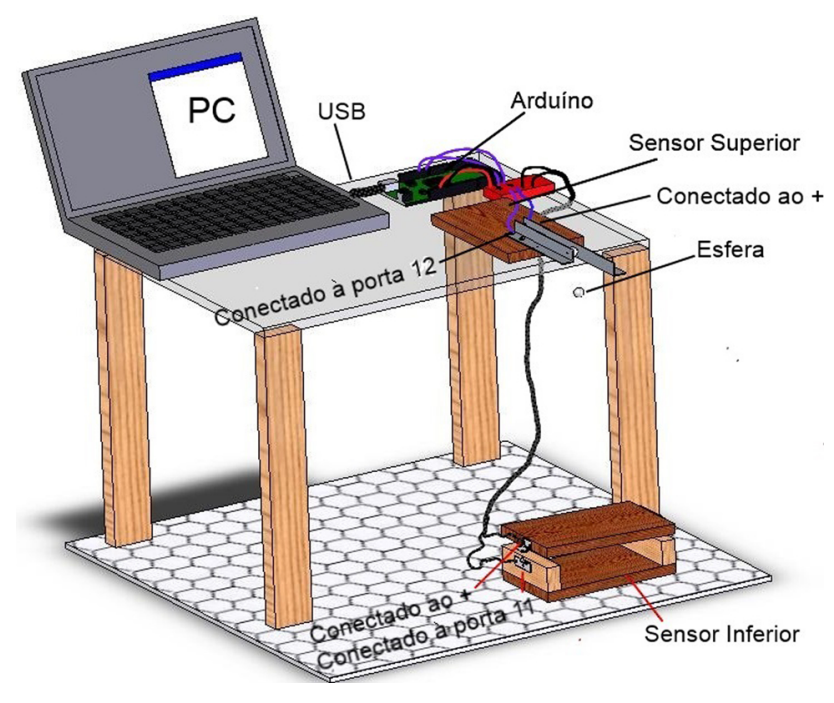

Figura 1: Visão geral do arranjo experimental.

a Figura 2, A alavanca móvel, presa à alavanca fixa por meio de um ou mais atilhos de borracha, pressiona a esferinha contra a alavanca fixa. Enquanto a esferinha de aço estiver presa e logo em contato com as alavancas, teremos uma voltagem de $5 \mathrm{~V}$ (nível alto) na porta 12 da placa Arduíno. Quando a alavanca móvel for manualmente empurrada para o lado e a esfera liberada, o circuito ficará aberto e a voltagem cai para 0V (nível baixo) na porta 12 . Depois de cair uma altura predeterminada $h$, a esfera atinge o sensor inferior - Figura 2-que consiste em uma base de madeira móvel ligeiramente inclinada que sob o efeito do impacto com a esfera aciona um contato elétrico levando a porta 11 do Arduíno para nível alto. Assim que a esfera rola para fora da base esta porta retorna ao nível baixo, veja o esquema da Figura 3 onde também é mostrado o circuito de apoio. O microcontrolador Arduíno registra o instante da liberação e do impacto, logo, a duração do tempo queda, depois permanece inativo durante 10 segundos antes de estar pronto para a próxima medida. A esfera é então recolocada na posição inicial levando o sensor superior novamente para o nível alto, permitindo uma nova medida. No sensor superior, há um furo de $1 \mathrm{~mm}$ de diâmetro onde a esfera deve ser encaixada, garantindo sempre o mesmo ponto de lançamento sem risco de mudança na altura. A altura medida é a distância entre o ponto de impacto da esfera no sensor inferior e a sua parte inferior, quando esta ainda está presa no sensor superior, assim o diâmetro da esfera não causa erros. A altura $h$ foi medida com uma trena metálica com divisões de $1 \mathrm{~mm}$. O espaço entre os
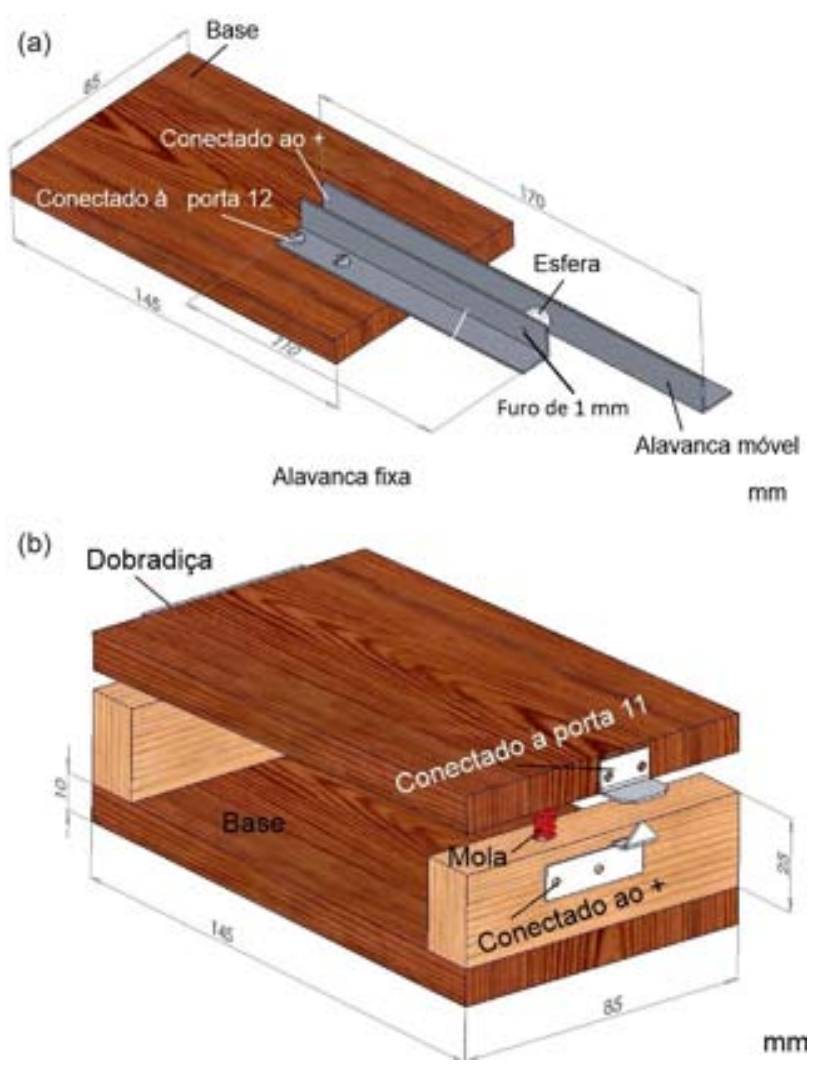

Figura 2: Sensores superior (a) e inferior (b). No desenho, por clareza, o espaço entre os contatos elétricos no sensor inferior está exagerado, no experimento ele é inferior a $1 \mathrm{~mm}$. $O$ peso da esfera ao atingir a base do sensor inferior permite que os contatos se toquem e ao rolar para fora da base, permite que a mola restaure a condição inicial.

contatos elétricos do sensor inferior é muito inferior a $1 \mathrm{~mm}$. Convém ressaltar que a esfera metálica, assim como os contatos metálicos dos sensores, devem estar perfeitamente limpos para evitar interrupções indevidas na comunicação com a placa Arduíno. O código-fonte para aquisição de dados com Arduino é mostrado no Apêndice. Para informações sobre a programação da placa Arduino, veja por exemplo, 5 e referências ali citadas.

\section{Resultados experimentais}

Para $h=0,943 \mathrm{~m} \operatorname{com} \delta h=0,001 \mathrm{~m}$ e $\delta t=0,001 \mathrm{~s}$, os tempos de queda vertical a partir do repouso são mostrados na Tabela 1.

Os valores obtidos para $g_{i}$ correspondem ao valor médio de cada medida obtido a partir da equação (1) da seguinte forma:

$$
\bar{g}_{i}=\frac{g_{i \mathrm{máx}}+g_{i \mathrm{~min}}}{2}
$$



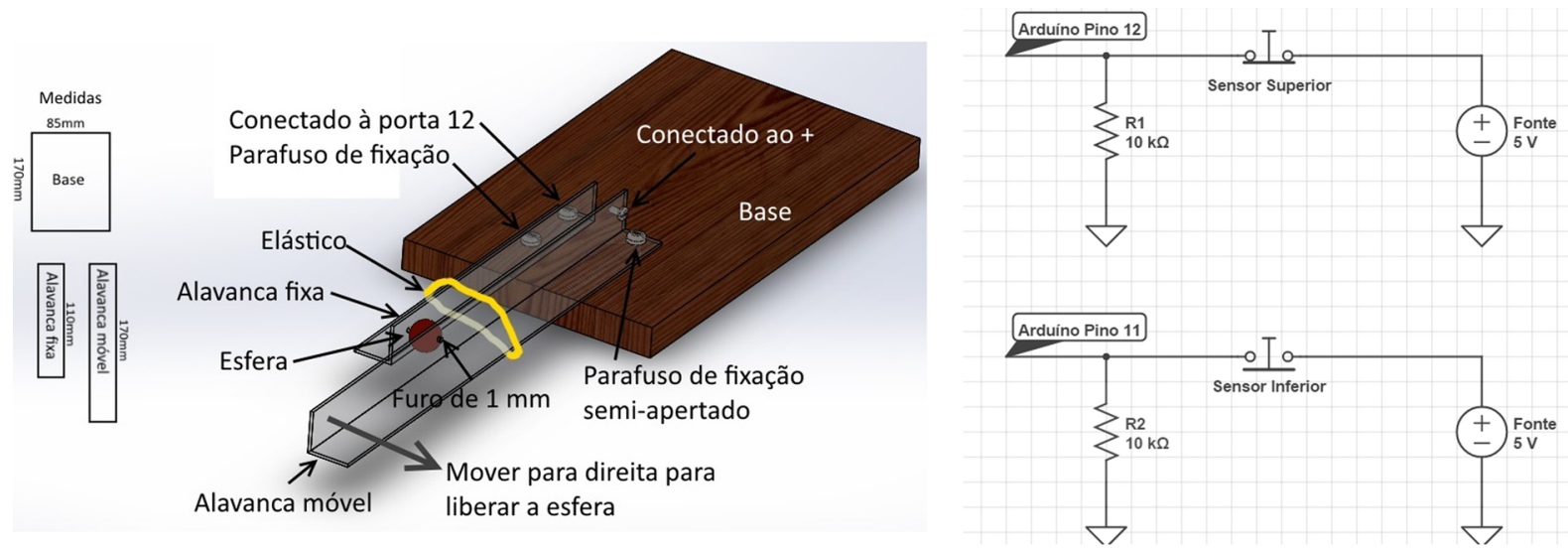

Figura 3: Detalhes do sensor superior e plataforma de lançamento. Note que a esferinha fica encaixada em um pequeno furo e ali mantida pela pressão mecânica gerada pelo atilho de borracha. A esferinha é liberada quando a alavanca móvel é empurrada lateralmente. Ao lado, o circuito de apoio.

Tabela 1: Medidas dos tempos de queda-livre. A duração média da queda é de $0,439 \mathrm{~s}$.

\begin{tabular}{lccccc}
\hline Med. & $t_{i}(\mathrm{~s})$ & Med. & $t_{i}(\mathrm{~s})$ & Med. & $t_{i}(\mathrm{~s})$ \\
\hline 1 & 0,439 & 8 & 0,439 & 15 & 0,439 \\
2 & 0,439 & 9 & 0,439 & 16 & 0,439 \\
3 & 0,440 & 10 & 0,440 & 17 & 0,439 \\
4 & 0,439 & 11 & 0,439 & 18 & 0,439 \\
5 & 0,439 & 12 & 0,440 & 19 & 0,439 \\
6 & 0,439 & 13 & 0,440 & 20 & 0,439 \\
7 & 0,438 & 14 & 0,440 & 21 & 0,439 \\
\hline
\end{tabular}

onde

$$
g_{i \text { máx }}=\frac{2(h+\delta h)}{\left(t_{i}-\delta t\right)^{2}}
$$

e

$$
g_{i \min }=\frac{2(h-\delta h)}{\left(t_{i}+\delta t\right)^{2}} .
$$

A incerteza individual $\delta g_{i}$ pode ser calculada com a expressão

$$
\delta g_{i}=\frac{g_{i \text { máx }}-g_{i \text { mín }}}{2},
$$

ou do modo usual [8]. O resultado é $\delta g_{i}=0,055$ $\mathrm{m} / \mathrm{s}^{2}$ para todas as vinte e uma medidas individuais. Os resultados para $\bar{g}_{i}$ são mostrados na Tabela 2. O valor médio de $\bar{g}_{i}$ é $9,778 \mathrm{~m} / \mathrm{s}^{2}$ e o desvio padrão da média ou erro padrão é $0,012 \mathrm{~m} / \mathrm{s}^{2}, \operatorname{logo}$ o experimento nos dá

$$
g_{\text {exp. }}=9,78 \pm 0,01 \mathrm{~m} / \mathrm{s}^{2} .
$$

O resultado dado pela Eq. (6) deve ser comparado com uma aproximação apropriada ao valor de $g$ local medido por Souza e Santos do Observatório Nacional [9] em 2010,
Tabela 2: Valores de $\bar{g}_{i}$; a incerteza de cada medida individual é $\delta g_{i}=\delta g=0,055 \mathrm{~m} / \mathrm{s}^{2}$.

\begin{tabular}{cccccc}
\hline Med. & $\bar{g}_{i}\left(\mathrm{~m} / \mathrm{s}^{2}\right)$ & Med. & $\bar{g}_{i}\left(\mathrm{~m} / \mathrm{s}^{2}\right)$ & Med. & $\bar{g}_{i}\left(\mathrm{~m} / \mathrm{s}^{2}\right)$ \\
\hline 1 & 9,786 & 8 & 9,786 & 15 & 9,786 \\
2 & 9,786 & 9 & 9,786 & 16 & 9,786 \\
3 & 9,742 & 10 & 9,742 & 17 & 9,786 \\
4 & 9,786 & 11 & 9,786 & 18 & 9,786 \\
5 & 9,786 & 12 & 9,742 & 19 & 9,786 \\
6 & 9,786 & 13 & 9,742 & 20 & 9,786 \\
7 & 9,831 & 14 & 9,742 & 21 & 9,786 \\
\hline
\end{tabular}

$$
g_{\text {Rio de Janeiro }}=978789,852 \pm 0,011 \mathrm{mGal},
$$

onde $1 \mathrm{mGal}=1 \times 10^{-5} \mathrm{~m} / \mathrm{s}^{2}$. Para fins de comparação com o resultado experimental obtido basta que consideremos o valor aproximado

$$
g_{\text {Rio. }} \approx 9,79 \mathrm{~m} / \mathrm{s}^{2} .
$$

O desvio percentual relativo é

$$
\frac{|9,79-9,78|}{9,79} \times 100 \approx 0,1 \% .
$$

Nosso resultado pode também ser comparado com os obtidos com outros métodos, veja por exemplo, as referências $[7,10-12]$. É possível medir também com o modelo de placa Arduino que utilizamos (Mega 2560) intervalos de tempo com precisão de microssegundos, mas, no caso do arranjo experimental proposto, os registros das durações temporais são instáveis e muitos dados devem ser descartados, o que nos parece inconveniente desde um ponto de vista pedagógico 


\section{Comentários finais}

O fator que realmente diferencia esta proposta experimental no nível do laboratório do ensino médio é a possibilidade de medir intervalos de tempo com precisão de milissegundos com um arranjo simples e de baixo custo. De fato, o custo total - sem contar o computador - pode ser igual ou menor do que $\mathrm{R} \$ 60$. Neste experimento usamos um microcontrolador Arduíno Mega, que pode ser substituído pelo Arduíno UNO de menor custo, sem necessidade de alteração na programação. Versões mais baratas dos microcontroladores Arduío originais, os Garaginos, podem ser encontradas por $\mathrm{R} \$ 45,00$ (a versão similar); a protoboard de 170 furos, custa por volta de $\mathrm{R} \$ 6,00$, e muitas vezes acompanha o kit Arduíno. Os dois resistores e os fios também são de baxíssimo custo. A base dos sensores foi feita com restos de madeira e as alavancas com sucata de alumínio. A mola que recoloca o sensor inferior na posição inicial foi tirada de uma impressora fora de uso.

Um dos autores do presente trabalho (H.C.) testou com sucesso o experimento com a colaboração voluntária dos alunos do terceiro ano do ensino médio de uma escola pública localizada no município do Rio de Janeiro. O experimento foi realizado no contraturno quando os alunos permanecem na escola sem a obrigação de estar em sala de aula. Como quase sempre acontece quando os alunos tem a oportunidade de "colocar a mão na massa", a resposta foi gratificante para o professor. Mas deve ser observado que não foi exigido dos alunos que programassem o microcontrolador. Isto foi feito pelo professor e deste modo os alunos puderam concentrar-se na física envolvida no experimento e no tratamento dos dados que procuramos fazer do modo mais simples possível. Em condições regulares, os autores estimam que duas aulas de 50 minutos seriam suficientes para que os alunos pudessem realizar com eficácia o experimento. Na primeira aula seriam apresentados os fundamentos teóricos e o arranjo experimental dando a oportunidade aos alunos de familiarizaremse com o processo da medida a ser feita. Na segunda aula, os alunos poderiam então colher os dados e analisá-los.

Mas, notemos que nada impede que havendo disponibilidade de tempo, rudimentos da programação do micontrolador Arduíno também possam ser introduzidos 1 A questão da introdução do ensino

\footnotetext{
${ }^{1} \mathrm{~A}$ programação do microcontrolador Arduíno é feita em $\mathrm{C}^{++}$.
}

de programação é contorversa, mas aqui, dada a simplicidade do experimento, ela é opcional.

Um experimento como este apresenta muitas possibilidades pedagógicas. Pode ser utilizado como uma experiência de laboratório de física básica em um curso formal, um projeto extraclasse para um grupo de alunos ou um projeto individual. Observe também que embora pensado para o laboratório básico nos níveis médio e universitário, este experimento pode ser realizado facilmente em casa por um aluno ou um amador interessado. Há uma comunidade Arduino formada por grupos que atuam nas redes sociais e que são totalmente dedicados à troca de experiências e informações envolvendo o uso e a programação do microcontrolador Arduíno e muitos de nossos alunos participam desses grupos.

\section{Apêndice: código de aquisição de dados com Arduino}

Por razões pedagógicas, o código-fonte utilizado é simples e procura obter apenas o tempo de duração da queda. Para maiores informações sobre a programação da placa Arduino ver [5,6].

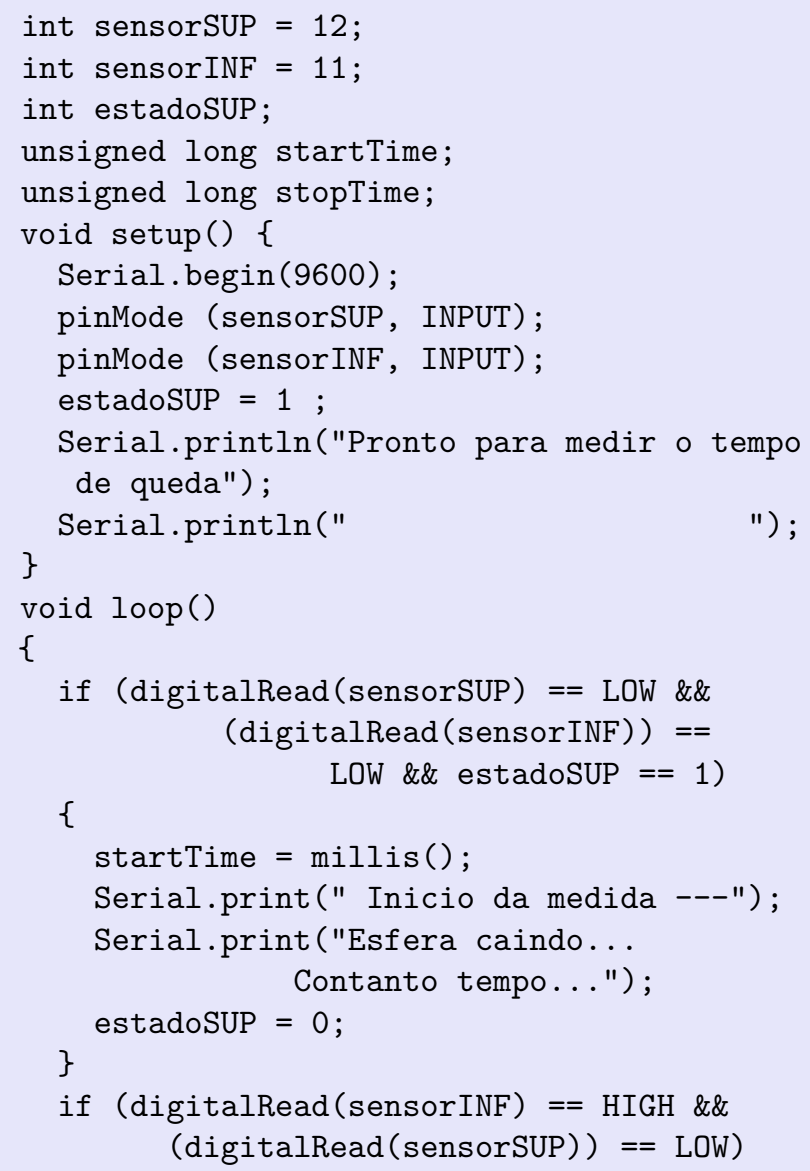




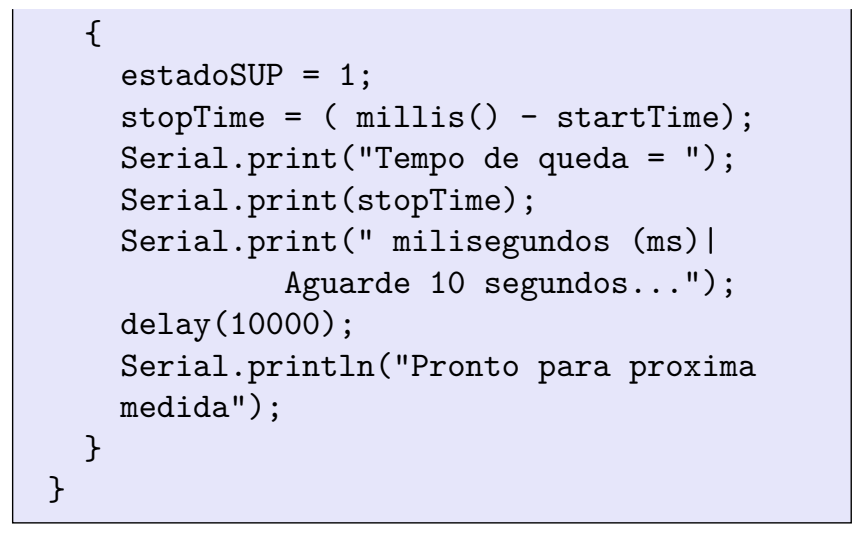

\section{Agradecimentos}

Os autores agradecem ao colega Dr. V. Soares pela leitura crítica do manuscrito original e aos árbitros pelas sugestões pertinentes.

\section{Referências}

[1] J.A. Blackburn and R. Koenig Am. J. Phys. 44, 855 (1976).

[2] M.A. Cavalcante, A. Bonizzia e L.C.P. Gomes, Rev. Bras. En. Fís. 30, 2501 (2008).

[3] M.A. Cavalcante, C.R.C. Tavolar e E. Molisani, Rev. Bras. En. Fís. 33, 4503 (2011).

[4] V.R. Coluci, G. Paulino, D.C. de Souza e E.P.R. Vasconcelos, Rev. Bras. En. Fís. 35, 2506 (2013).

[5] A.R. de Souza, A.C. Paixão, D.D. Uzêda, M.A. Dias, S. Duarte e H.S. de Amorim, Rev Bras. En. Fís. 33, 1702 (2011).

[6] H S. Amorim, M.A. Dias e V. Soares, Rev. Bras. En. Fís. 37, 4310 (2015).

[7] C.E. Aguiar e F. Laudares, Am. J. Phys. 71, 499 (2003).

[8] J.G. Taylor, An Introduction to Error Analysis (University Science Books, Boulder, 1982), 2nd ed.

[9] M.A. de Souza e A.A. dos Santos Absolute gravimetry on the Agulhas Negras calibration line, Rev. Bras. Geof. 28, 165 (2010).

[10] J.A. White, A. Medina, F.L. Román and S. Velasco, Phys. Teach. 45175 (2007)

[11] R. De Luca e S. Ganci Rev. Bras. En. Fís. 33, 3301 (2011).

[12] O. Schwarz, P. Vogt e J. Kunh, Phys. Teach. 51312 (2013). 